
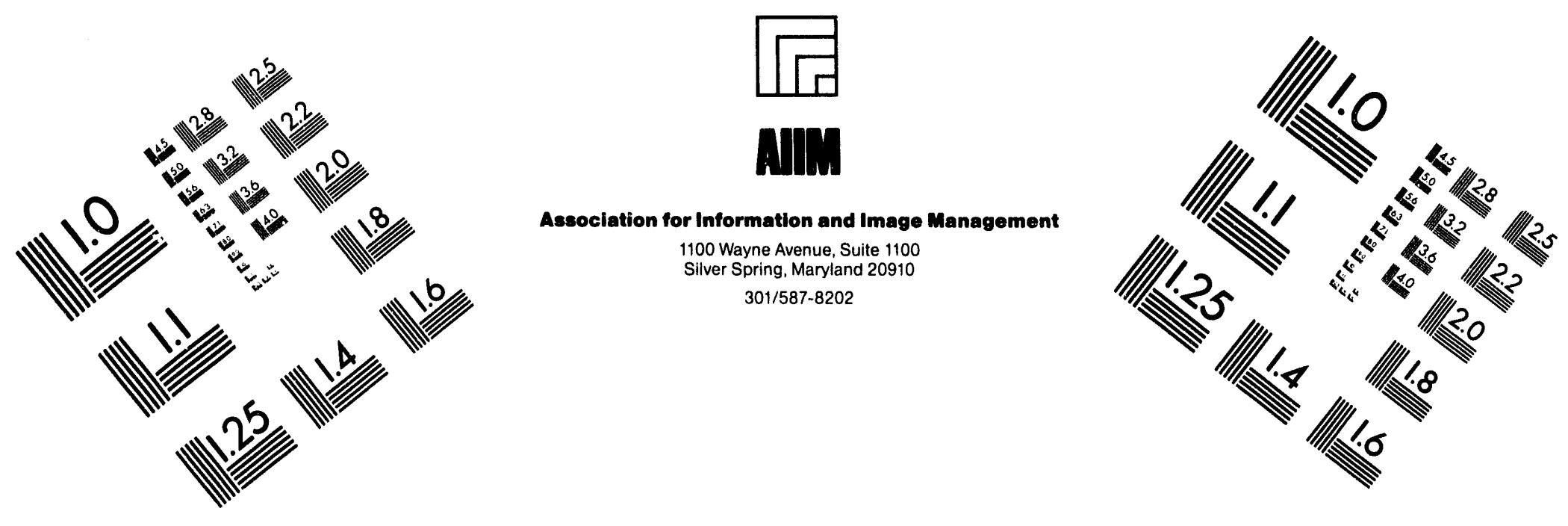

\title{
Centimeter
}

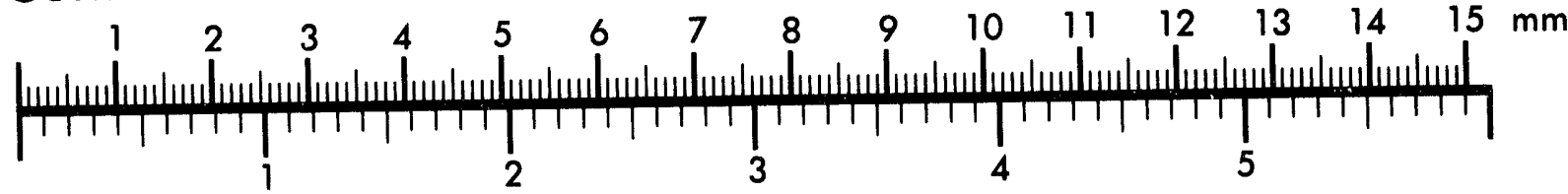
Inches
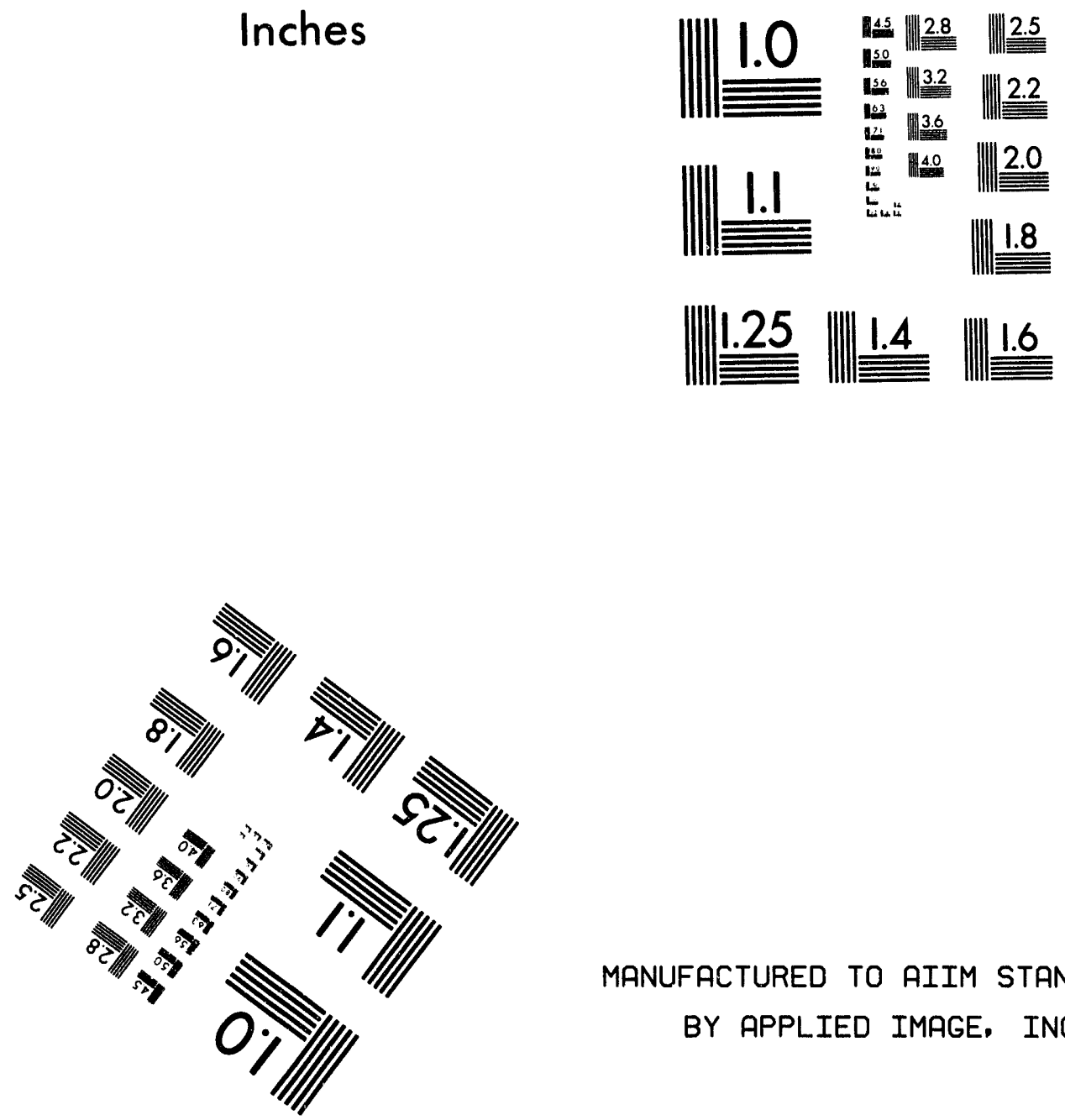

MANUFACTURED TO AIIM STANDARDS

BY APPLIED IMAGE, INC.

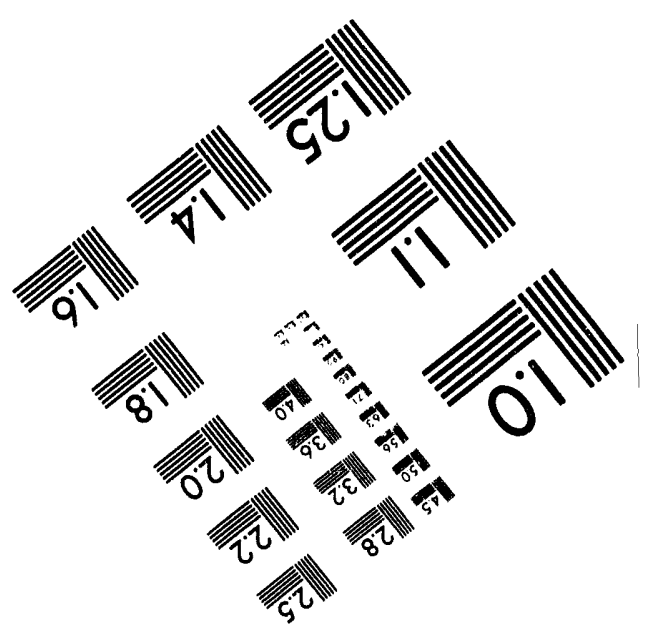



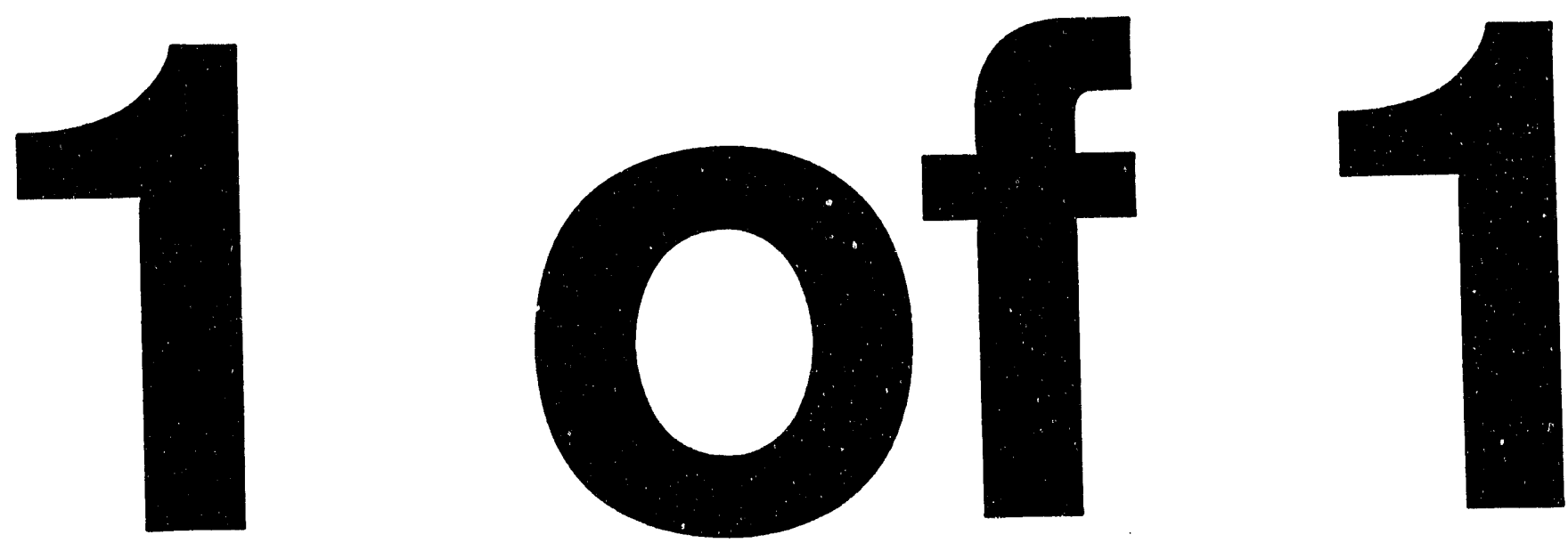
PNL-SA-23097

\section{BETA DECAY DATA FOR NEUTRON-RICH LI-CL NUCLIDES}

P. L. Reeder et al

May 1994

Presented at the

International Conference on Nuclear

Data for Science and Technology

May 9-13, 1994

Gatlinburg, Tennessee

Prepared for

the U.S. Department of Energy

under Contract DE-AC06-76RLO 1830

\section{DIBTRIBUTION OF THIS DOCUMENT IS UNLIMITED}

Pacific Northwest Laboratory

Richland, Washington 99352

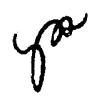

\section{DISCLAIMER}

This report was prepared as an account of work sponsored by an agency of the Uniter States Government. Neither the United States Government nor any agency thereof, nor any of their employees, makes any warranty, express or implied, or assumes any legal liability or responsibility for the accuracy, completeness, or usefulness of any information, apparatus, product, or process disclosed, or represents that its use would not infringe privately owned rights. Reference herein to any specific commercial product, process, or service by trade name, trademark, manufacturer, or otherwise does not necessarily constitute or imply its endorsement, recommendation, or favoring by the United States Government or any agency thereof. The views and opinions of authors expressed herein do not necessarily state or reflect those of the United States Government or any agency thereof. 


\section{BETA DECAY DATA FOR NEUTRON-RICH LI-CL NUCLIDES}

\author{
P. L. Reeder \\ Y. Kim \\ W. K. Hensley \\ H. S. Miley \\ R. A. Warner \\ Pacific Northwest Laboratory \\ Richland, WA 99352 \\ 509-376-0948
}

\author{
D. J. Vieira \\ J. M. Wouters \\ Z. Y. Zhou \\ H. L. Seifert \\ Los Alames National Laboratory \\ Los Alamos, NM 87545 \\ 505-667-7231
}

\begin{abstract}
We have used the Time-of-Flight Isochronous (TOFI) spectrometer at the LAMPF accelerator to systematically measure the half-life $\left(t_{1 / 2}\right)$, delayed neutron emission probability $\left(P_{n}\right)$, beta-delayed multi-neutron emission, average energy of delayed neutron spectra, and betadelayed alpha emission for a large number of neutron-rich isotopes for all $\mathrm{Z}$ values between $\mathrm{Li}$ and $\mathrm{Cl}$. The TOFI spectrometer measures the $A, Z$, and $Q$ of each neutronrich ion and determines the decay properties by means of a delayed coincidence between each ion and subsequent decay products.
\end{abstract}

\section{INTRODUCTION}

Measurements of the half-life $\left(t_{1 / 2}\right)$, delayed neutron emission probability $\left(P_{n}\right)$, beta-delayed multi-neutron emission $\left(P_{2 n}, P_{3 n}\right.$, etc.), average energy of delayed neutron spectra, and beta-delayed alpha emission are important for understanding the nuclear decay properties of neutron-rich nuclei, improving models for astrophysical applications and delineating half-life systematics. The large decay energies (e.g., 10-20 MeV) that arise in these nuclei because of the increasingly asymmetric proton to neutron ratios open up many new decay channels such as beta-delayed multi-neutron emission and beta-delayed charged-particle emission. By measuring masses, halflives, $P_{n}$, etc., a detailed comparison can be made to various predictions calculated from the shell or QRPA models. For fission-product precursors, one can correlate shorter half-lives and smaller $P_{n}$ values with beta strength predominantly populating low-lying, neutron-bound states, whereas longer half-lives and higher $P_{n}$ values are associated with beta strength predominantly feeding highlying, neutron-unbound states. These generalizations may not apply in the light-mass precursors studied in this work because of the much lower density of states and rapidly changing nuclear structure.

The Time-of-Flight Isochronous (TOFI) spectrometer at LAMPF analyzes fragmentation products in the light mass region produced by $800 \mathrm{MeV}$ proton bombardment of a ${ }^{232} \mathrm{Th}$ target. The TOFI spectrometer was originally built to measure nuclidic masses by means of Time-ofFlight. 1,2 It can identify recoiling ions according to their mass $(A)$, nuclidic charge $(Z)$, and ionic charge $(Q)$, but does not physically separate the recoil nuclides at its focal point. The LAMPF proton beam is pulsed with a repetition rate of 120 pulses per second. Ions are detected during the beam pulse, and beta-decay products are detected during the 7-ms period between beam pulses.

\section{EXPERIMENTS}

In our decay experiments, the ions were deposited in a thin Si detector located at the final focus of the TOFI spectrometer. This thin Si detector was surrounded by a 2mm-thick plastic scintillator cup plus a thick Si detector which together were used to measure beta particles. The vacuum pipe containing these detectors was surrounded by a high-efficiency neutron counter. By counting neutrons in addition to betas, we could preferentially measure the lowyield very neutron-rich nuclides rather than the abundant beta/gamma emitters produced close to the valley of stability. For half-lives less than about $0.5 \mathrm{~s}$, beta counting provided a useful measure of the half-lives. In addition, beta-neutron coincidence counting gave an alternative means of measuring the delayed-neutron emission probabilities.

Decay information was obtained from a delayedcoincidence technique in which the arrival times of each identified ion and each decay particle were recorded. Histograms of time intervals between ion arrival and detection of the decay products were constructed off-line by software for each ion type. These histograms included an exponential component corresponding to the correlated decay events and a flat-background component due to random correlations. A least-squares fitting program was used to determine the slope and intercept of the exponential component. The program included corrections for decay of daughter and grand-daughter components whenever such corrections were necessary.

The half-lives reported here are based on a weighted average of the half-lives obtained from beta, neutron, and beta-neutron-coincidence time-interval histograms. 
Delayed neutron emission probabilities $\left(P_{n}\right)$ were determined by two techniques-one inv lving the abundance of neutrons relative to the number of ions and the other involving the abundance of neutrons relative to the number of betas. Results for $P_{n}$ values are based on the weighted average of these two techniques.

The neutron counter consisted of $44{ }^{3} \mathrm{He}$ propertional counter tubes embedded in a polyethylene moderator. These tubes were arranged in three concentric rings. Because the mean path length of a neutron in the moderator is a function of neutron energy, we used the ratio of counts in the outer ring to the inner ring to determine the average energy of the delayed-neutron spectrum. ${ }^{3}$ Average energies from experimentally -measured energy spectra for delayed neutrons from ${ }^{9} \mathrm{Li}$, ${ }^{15} \mathrm{~B}$, and ${ }^{16} \mathrm{C}$ were ssed to construct a calibration curve of ring ratio versus average energy. The ring ratios were also used to correct the neutron-counter efficiency used in the $P_{n}$ calculation for the slight energy dependence of the neutron counter.

Beta-delayed multi-neutron emission could also be detected. The residence time for neutrons in our counter is about $37 \mu \mathrm{s}$. If tw/o or more neutrons are detected within a $200 \mu \mathrm{s}$ window, we classified the event as a multi-neutron event. Decay curves for multi-neutron events were created in the same manner as for single-neutron events. By fitting multi-neutron decay curves for specific nuclides, we could determine the probabilities for $\mathrm{P}_{2 \mathrm{n}}, \mathrm{P}_{3 \mathrm{n}}$, etc.

In our recent experiments, we used separate preamplifiers and amplifiers on each of the 8 tubes in the inner ring. Thus we could determine the angular correlation for two-neutron events. The goal was to determine the mechanism for two-neutron emission. A measurement of the angular correlation between the two neutrons should distinguish the sequential-emission mechanism which has a uniform angular correlation and the simultaneous emission of a di-neutron, which has a forward-peaked angular distribution. The analysis of these data is still in progress.

Ions passing through the TOFI spectrometer were implanted in the thin Si detector located at the focal point. During beam-on periods, the $\mathrm{Si}$ detector measured the energy of the ions. Between beam bursts, the same $\mathrm{Si}$ detector measured the energy of any charged particles emitted during beta decay. By correlating the pulse height spectrum in the thin Si detector with specific ions, we could search for beta-delayed deuteron, triton, or alpha emission.

\section{RESULTS}

Results from our first experiment done in 1987 on the $\mathrm{Li}$ to $\mathrm{Al}$ nuclides have been published. ${ }^{4}$ We reported halflives for $\mathbf{1 8}$ nuclides and neutron-emission probabilities for
19 nuclides. The half-lives of $25 \mathrm{~F}$ and ${ }^{28} \mathrm{Ne}$ were measured for the first time. Delayed neutron emission was observed for the first time from ${ }^{12} \mathrm{Be},{ }^{14} \mathrm{~B},{ }^{17} \mathrm{C},{ }^{18} \mathrm{~N},{ }^{25} \mathrm{~F}$, and ${ }^{28} \mathrm{Ne}$.

In 1992, we repeated our measurements in the $\mathrm{Li}$ to $\mathrm{F}$ region with an emphasis on the extremely neutron-rich nuclides. The new data (1992) gave decay results for ${ }^{11} \mathrm{Li}$, ${ }^{14} \mathrm{Be},{ }^{17} \mathrm{~B},{ }^{20} \mathrm{C}$, and ${ }^{22} \mathrm{~N}$ and improved data for the other neutron-rich nuclides. ${ }^{5}$ A partial listing of the 1992 results is given in Table 1 . For ${ }^{17} \mathrm{C},{ }^{18} \mathrm{C}$, and ${ }^{18} \mathrm{~N}$, our $\mathrm{P}_{\mathrm{n}}$ values are significantly higher and our average energies are significantly lower than the values recently measured at Michigan State University. ${ }^{6}$ These discrepancies are probably explained by the fact that our neutron counter was sensitive to all neutron energies, including thermal energies, whereas the MSU/Notre Dame time-of-flight detector was not sensitive below about $0.8 \mathrm{MeV}$.

The beta-delayed charged-particle data were dominated by alphas from implanted ${ }^{8} \mathrm{Li}$ and ${ }^{9} \mathrm{Li}$. However, well known alpha peaks from ${ }^{18} \mathrm{~N}$ were clearly seen, and preliminary evidence for beta-delayed alpha emission from ${ }^{14} \mathrm{~B}$ was observed. In 1993, we took extensive data in the $\mathrm{F}$ to $\mathrm{Cl}$ mass resion. Analysis has begun on this data set, but only preliminary results are available.

\section{ACKNOWLEDGMENTS}

Pacific Northwest Laboratory is operated for the U.S. Department of Energy by Battelle Memorial Institute under contract DE-AC06-76RLO 1830. This work was performed under the auspices of the U.S. Department of Energy.

\section{REFERENCES}

1. J. M. Wouters, R. H. Kraus, Jr., D. J. Vieira, G. W. Butler, and K. E. G. Löbner, Z. Phys. A 331, 229 (1988);X. L. Tu et al., ibid. 337. 361 (1990).

2. D. J. Vieira, J. M. Wouters, K. Vaziri, R. H. Kraus. Jr., H. Wollnik, G. W. Butler, F. K. Wohn, and A. H. Wapstra, Phys. Rev. Lett. 57, 3253 (1986); X. G. Zhou, et al., Phys. Lett. B 260, 285 (1991).

3. P. L. Reeder, H. S. Miley, W. K. Hensley, R. A. Warner, H. L. Siefert, D. J. Vieira, J. M. Wouters, and Z. Y. Zhou, Proc. 6th Int. Conf. on Nuclei Far From Stability, Berncastel-Kues, Germany, July 19-24, 1992, Inst. Phys. Conf. Ser. No. 132, IOP Publishing, Bristol and Philadelphia, 1993.

4. P. L. Reeder, R. A. Warner, W. K. Hensley, D. J. Vieira, and J. M. Wouters, Phys. Rev. C 44, 1435 (1991). 5. Y.-K. Kim, "Measurement of the Half-Life, DelayedNeutron Emission Probability, Delayed-Neutron Average Energy, and Delayed Charged-Particle Energy Spectrum 
for Very Neutron-Rich Helium Through Sodium Nuclides," Ph.D. Thesis, Utah State University June, 1994. 6. K. W. Scheller, "The $\beta$ - Delayed Neutron Decay of the Exotic Nuclei ${ }^{18} \mathrm{~N},{ }^{17} \mathrm{C}$, and ${ }^{18} \mathrm{C} "$. Ph.D. Thesis, Notre Dame University, Nov., 1993.

TABLE I. Half-lives, delayed-neutron emission probabilities, and average neutron energies from 1992 TOFI experiment.

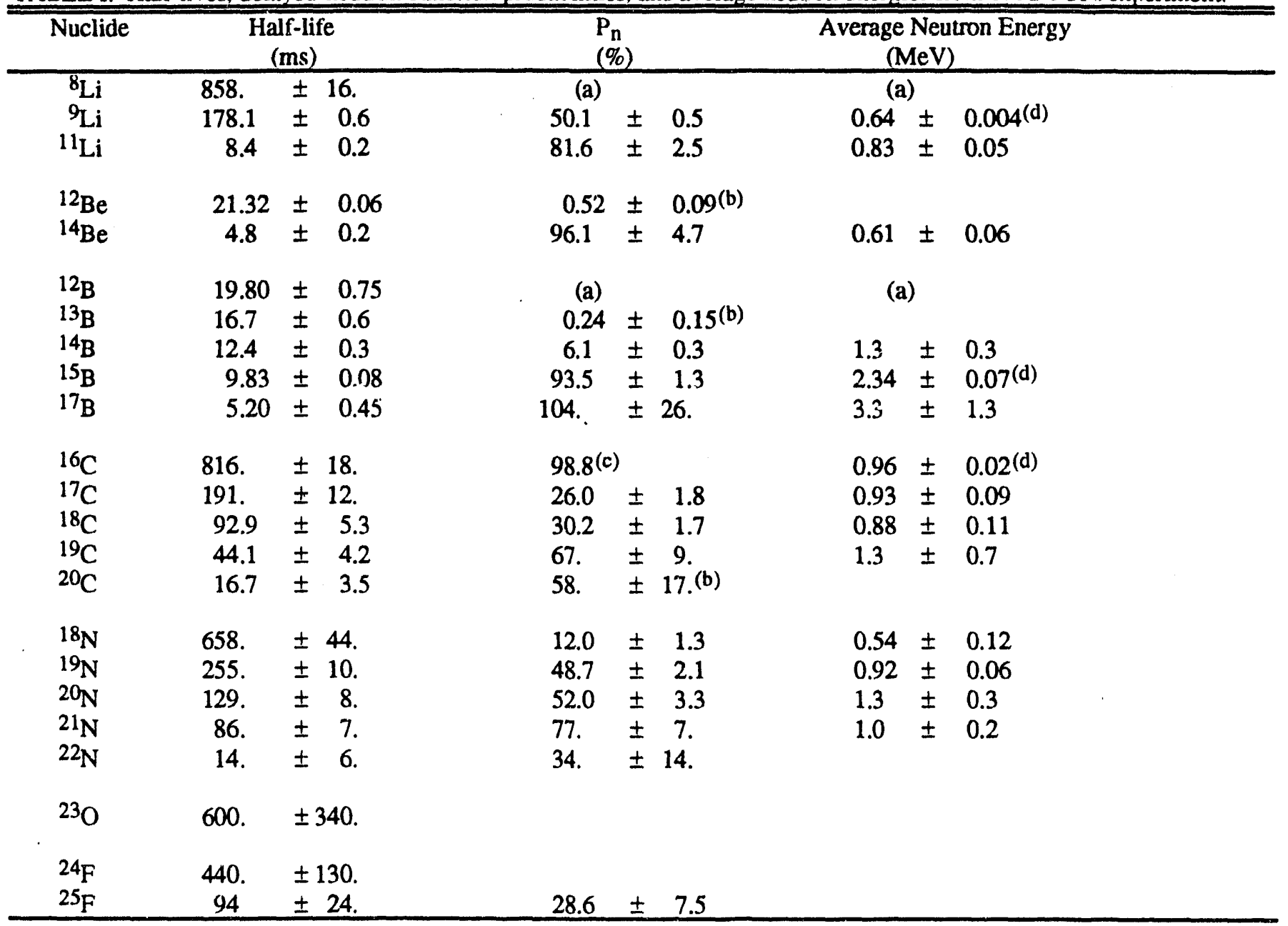

a Not a delayed neutron precursor.

b The neutron counter efficiency was assumed to be the same as for ${ }^{16} \mathrm{C}$. The uncertainty on the neutron counting efficiency was increased to $\pm 2.6 \%$.

${ }^{c}$ The literature $P_{n}$ value for ${ }^{16} \mathrm{C}$ was used to determine the effective neutron counting efficiency $(=54.8 \pm 0.3 \%)$.

d Used to determine calibration curve of Ring Ratio vs Average Energy. 

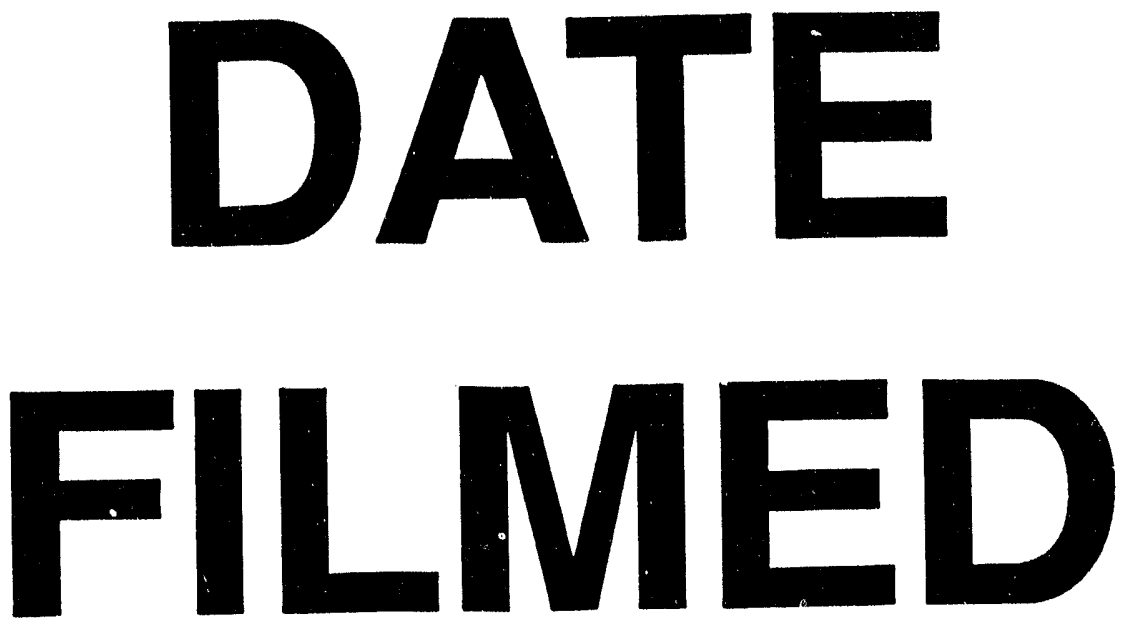

$8 / 11 / 94$
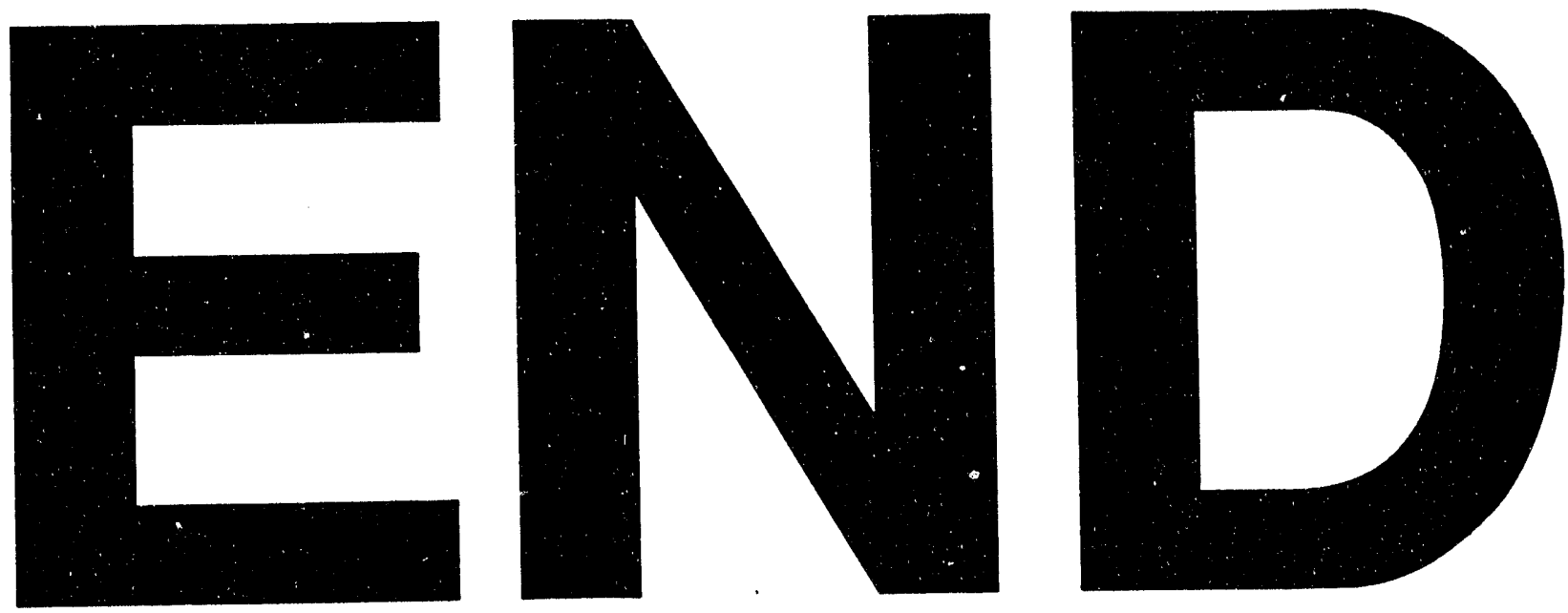
$\mid$ 